\title{
Chart-Based Transfer Rule Application in Machine Translation
}

\author{
Adam Meyers \\ New York University \\ meyers@cs.nyu.edu
}

\author{
Michiko Kosaka \\ Monmouth University \\ kosaka@monmouth.edu
}

\author{
Ralph Grishman \\ New York University \\ grishman@cs.nyu.edu
}

\begin{abstract}
Thansfer-based Machine Translation systems require a procedure for choosing the set of transfer rules for generating a target language translation from a given source language sentence. In an MT system with many competing transfor rules, choosing the best set of transfer rules for translation may involve the evaluation of an explosive number of competing sets. We propose a solution to this problem based on current bestfirst chart parsing algorithms.
\end{abstract}

\section{Introduction}

Transfer-based Machine 'Translation systems require a procedure for choosing the set of transfer rules for generating a target language translation from a given source language sentence. This procedure is trivial for a system if, given a context, one transfer rule can be selected unambiguously. Otherwise, choosing the best set of transfer rules may involve the evaluation of numerous competing sets. In fact, the number of possible transfer rule combinations incroases exponentially with the length of the source language sentence. This situation mirrors the problem of choosing productions in a nondeterministic parser. In this paper, we describe a system for choosing transfer rules, based on statistical chart parsing (Bobrow, 1990; Chitrao and Grishman, 1990; Caraballo and Charniak, 1997; Charniak ot al., 1998).

In our Machine Translation system, transfer rules are generated automatically from parsed parallel text along the lines of (Matsumoto et al., 1993; Meyers et al., 1996; Meycrs et al., 1998b). Our system tends to acquire a large number of transfer rules, due mainly to alternative ways of translating the same sequences of words, non-literal translations in parallel text and parsing errors. It is therefore crucial that our system choose the best set of rules efficiently. While the technique discussed here obviously applies to similar such systems, it could also apply to hand-coded systems in which each word or group of words is related to more than one transfer rule. For example, both Multra (Hein, 1996) and the Eurotra system described in (Way et al., 1997) require components for deciding which combination of transfer rules to use. The proposed technique may be used with systems like these provided that all transfer rules are assigned initial scores rating their appropriateness for translation. These appropriateness ratings could be dependent or independent of context. ${ }^{1}$

\section{Previous Work}

The MT literature describes several techniques for deriving the appropriate translation. Statistical systems that do not incorporate linguistic analysis (Brown ot al., 1993) typically choose the most likely translation based on a statistical model, i.c.., translation probability determines the translation. (Hein, 1996) reports a set of (hand-coded) feature structure based preference rules to choose annong alternatives in Multra. There is some discussion about adding some transfer rules automatically acquired from corpora to Multra. ${ }^{2}$ Assuming that they overgenerate rules (as we did), a system like the one we propose should be beneficial. In (Way et al., 1997), many different criteria are used to choose transfer rules to execute including: preferences for specific rules over gencral ones, and complex rule notation thati insures that few rules can apply to the same set of words.

The Pangloss Mark III system (Nirenburg

\footnotetext{
${ }^{1}$ This translation procedure would probably complement, not replace existing procedures in these systems.

${ }^{2}$ http://stp.ling.uu.se/ $\sim$ corpora/plug/reports/ ansk last/ is a report on this project for Multra.
} 
and Frederking, 1995) uses a chart-walk algorithm to combine the results of three MT engines: an example-based engine, a knowledgebased engine, and a lexical-transfer engine. Each engine contributes its best edges and the chart-walk algorithm uses dynamic programming to find the combination of edges with the best overall score that covers the input string. Scores of edges are normalized so that the scores from the different engines are comparable and weighted to favor engines which tend to produce better results. Pangloss's algorithm combines whole MT systems. In contrast, our algorith$\mathrm{m}$ combines output of individual transfer rules within a single MT system. Also, we use a bestfirst search that incorporates a probabilisticbased figure of merit, whereas Pangloss uses an empirically based wcighting scheme and what appears to be a top-down search.

Best-first probabilistic chart parsers (Bobrow, 1990; Chitrao and Grishman, 1990; Caraballo and Charniak, 1997; Charniak et al., 1998) strive to find the best parse, without exhaustively trying all possible productions. A probabilistic figure of merit (Caraballo and Charniak, 1997; Charniak et al., 1998) is devised for ranking edges. The highest ranking edges are pursued first and the parser halts after it produces a complete parse. We propose an algorithm for choosing and applying transfer rules based on probability. Each final translation is derived from a specific set of transfer rules. If the procedure immediately sclected these transfer rules and applied them in the correct order, we would arrive at the final translation while creating the minimum number of edges. Our procedure uses about 4 times this minimum number of edges. With respect to chart parsing, (Charniak ot al., 1998) report that their parser can achieve good results while producing about three times the minimum number of edges required to produce the final parse.

\section{Test Data}

We conducted two experiments. For experimen$\mathrm{t} 1$, we parsed a sentence-aligned pair of Spanish and English corpora, each containing 1155 sentences of Microsoft Excel Help Text. These pairs of parsed sentences were divided into distinct training and test sets, ninety percent for training and ten percent for test. The training

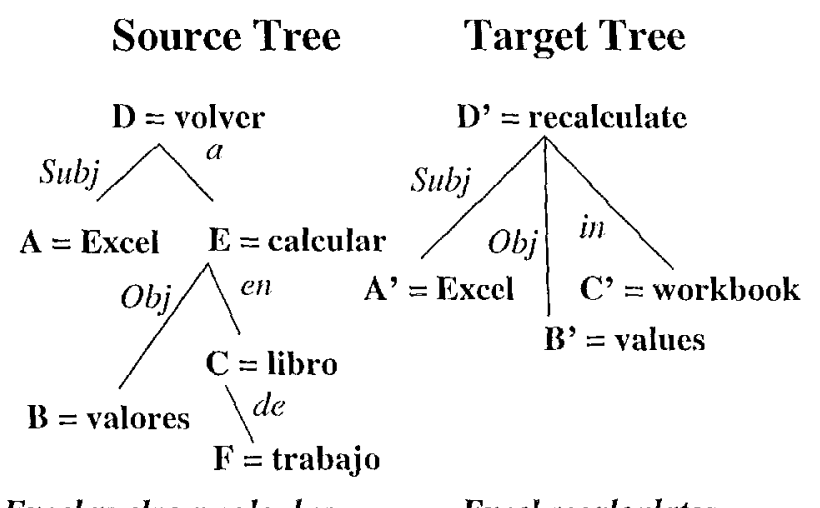

Excel vuelve a calcular valores en libro de trabajo

Figure 1: Spanish and English Regularized Parse Trees

set was used to acquire transfer rules (Meyers et al., 1998b) which were then used to translate the sentences in the test set. This paper focuses on our technique for applying these transfer rules in order to translatic the test sentences.

The test and training sets in experiment1 were rotated, assigning a different tenth of the sentences to the test set in each rotation. In this way we tested the program on the entire corpus. Only one test set (one tenth of the corpus) was used for tuning the system during development. Transfer rules, 11.09 on average, were acquired from each training set and used for translation of the corresponding test set. For Experiment 2, wo parsed 2617 pairs of aligned sentonces and used the same rotation procedure for dividing test and training corpora. The Experiment 2 corpus included the experiment 1 corpus. An avcrage of 2191 transfer rules were acquired from a given set of Experiment 2 training sentences.

Experiment 1 is orchestrated in a careful manner that may not be practical for extremcly large corpora, and Experiment 2 shows how the program performs if we scale up and climinate some of the fine-tuning. Apart from corpus size, there are two main difference between the two cxperiments: (1) the experiment1 corpus was aligned completely by hand, whereas the Experiment 2 corpus was aligned automatically using the system described in (Meyers et al., 1998a); and (2) the parsers were tuned to the experiment 1 sentences, but not the Experiment 2 sentences (that did not overlap with experiment1). 
1) $\Lambda=$ Excel

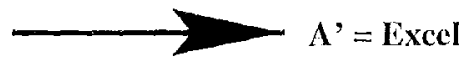

2) $\mathbf{B}=$ valores

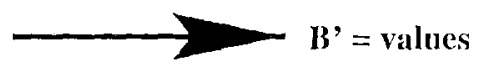

C = libro

3)

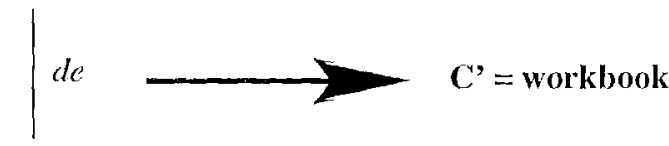

$F=$ trabajo

4)

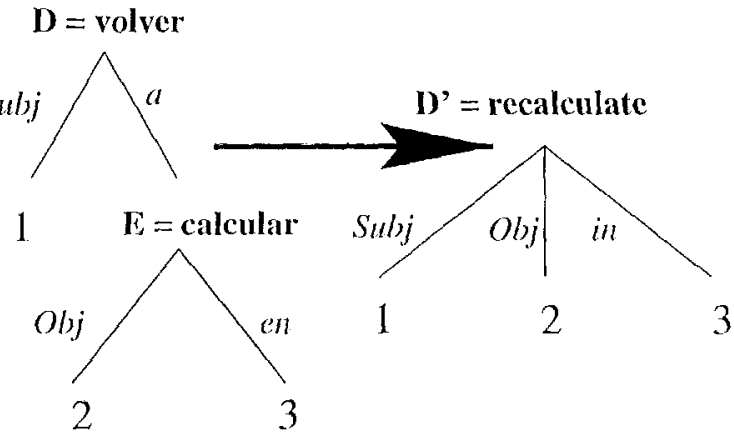

Figure 2: $A$ Set of Transfer Rules

\section{Parses and Transfer Rules}

Figure 1 is a pair of "regularized" parses for a corresponding pair of Spanish and Jnglish sentences from Microsoft Excel help text. These are F-structure-like dependency analyses of sentences that represent predicate argument structure. 'This representation serves to neutralize some differences between related sentence types, e.g., the regularized parse of related active and passive sentences are identical, except for the feature value pair $\{$ Mood, Passive . Nodes (valnes) aro labeled with head words and arcs (features) are labeled with grammatical functions (subject, object), prepositions (in) and subordinate conjunctions (before). ${ }^{3}$. For demonstration purposes, the source tree in Figure 1 is the input to our translation system and the target tree is the output.

The transfer rules in Figure 2 can be used to convert the input tree into the output tree. These transfer rules are pairs of corresponding rooted substiuctures, where a substructure (Matsumoto et al., 1993) is a connectiod set of arcs and nodes. A rulc

\footnotetext{
${ }^{3}$ Morphological features and their values (GramNumber: plural) are also represented as arcs and nodes.
}

consists of cither a pair of "open" substructures (rule 4) or a pair of "closed" substiructures (rules 1,2 and 3 ). Closed substructures consist of $\mathrm{s}$ ingle nodes $\left(A, \Lambda^{\prime}, B, B^{\prime}, C^{\prime}\right)$ or subtrees (the loft hand side of rule 3 ). Open substructures contain one or more open arcs, arcs without heads (bothl substructuros in rule 4 ).

\section{Simplified Translation with Tree-based Transfer Rules}

The rules in Figure 2 could combine by filling in the open arcs in rule 4 with the roots of the substructures in rules 1,2 and 3 . The result would be a closed edge which maps the left treo in Figure 1 into the right tree. Just as edges of a chart parser are based on the context free rules used by the chart, parser, edges of our translation system are based on these transfer rules. Initial edgos are identical to transfer rules. Other edges result from combining one closed edge with one open edge. Figure 3 lists the sequence of edges which would result from combining the initial edges based on Rules $1-4$ to replicate tho trees in Figure 1. The translation proceeds by incrementally matching the left hand sides of Rules 1-4 with the iuput tree (and insuring that the tiree is completely covered by these rules). The right-hand sides of these compatible rules are also combined to produce the translation. This is an idealized view of our system in which. cach node in the iuput tree matches the lefthand side of exactly one transfer rule: there is no ambiguity and no combinatorial explosion. The reality is that more than one transfer rules may bo activated for each node, as suggested in Figure 4.4 If each of the six nodes of the source tree corresponded to five transfer rules, there are $5^{6}=15625$ possible combinations of rules to consider. To produce the output in Figure 3 , a minimum of seven edges would be required: four initial edges derived from the original transfer rules plus three additional edges representing the combination of edges (steps 2 , 3 and 4 in Figure 3 ). The speed of our system is measured by the number of actual edges divided by this minimum.

\footnotetext{
${ }^{4}$ The third example listed would actually involve two transfer rules, one translating "volver" to "repeat" and the second translating "calcular" to "calculate".
} 
1)
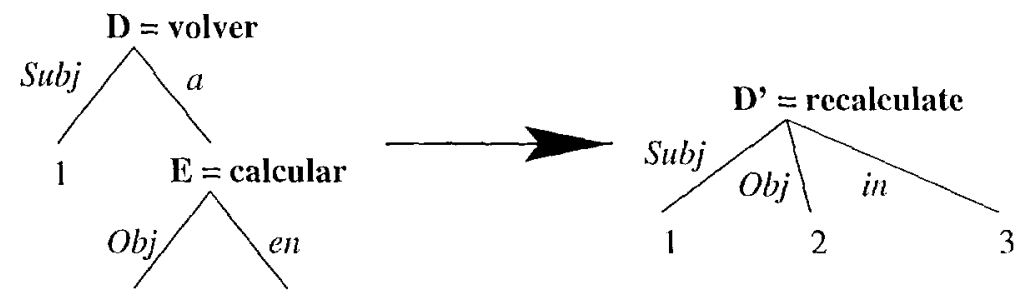

2)

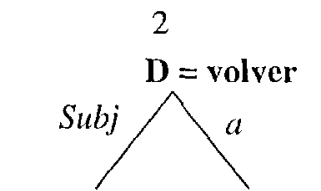

3

)
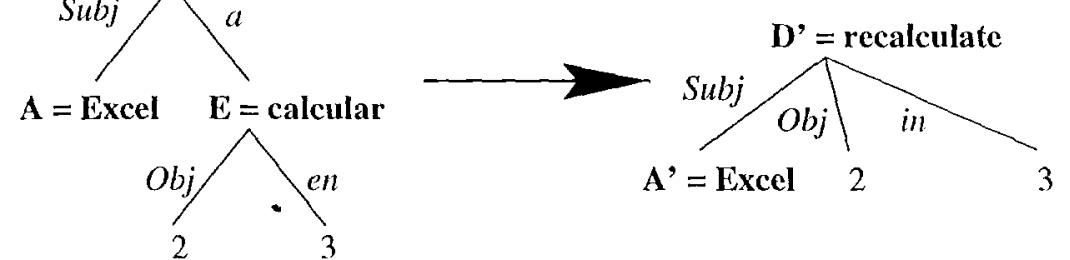

3)
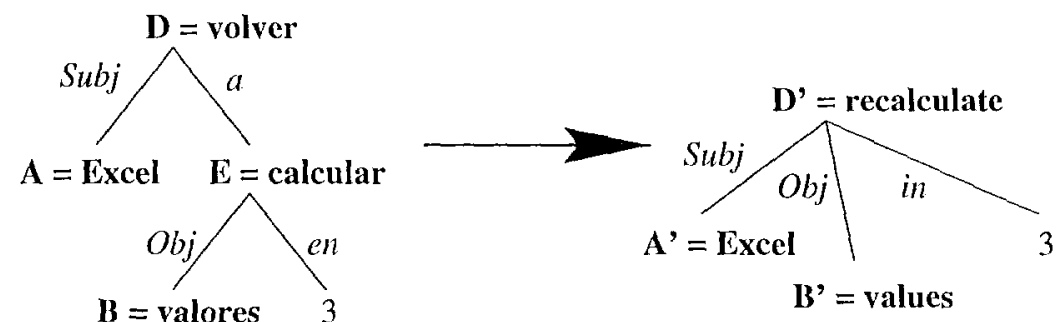

4)
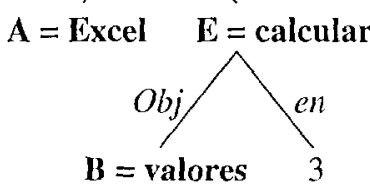

$\mathbf{B}^{\prime}$ = values

$\mathrm{D}=$ volver

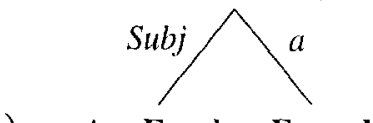

$\mathbf{A}=$ Excel $\quad \mathbf{E}=$ calcular
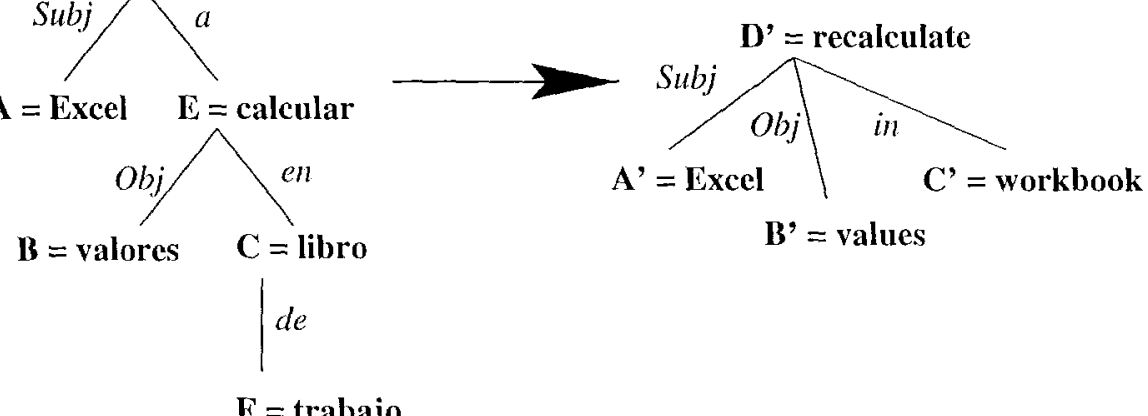

Figure 3: An Idealized Translation Procedure

\section{Best First Translation Procedure}

The following is an outline of our best first search procedure for finding a single translation:

1. For each node $N$, find $T_{N}$, the set of compatible transfer rules

2. Create initial edges for all $T_{N}$

3. Repeat until a "finished" edge is found or an edge limit is reached:

(a) Find the highest scoring edge $E$ (b) If complete, combine $E$ with compatible incomplete edges

(c) If incomplete, combine $E$ with compatible complete edges

(d) Incomplete edge + complete edge $=$ new edge

The procedure creates one initial edge for each matching transfer rule in the database $^{5}$ and puts these edges in a

\footnotetext{
${ }^{5}$ The left-hand side of a matching transfer rule is compatible with a substructure in the input source tree.
} 

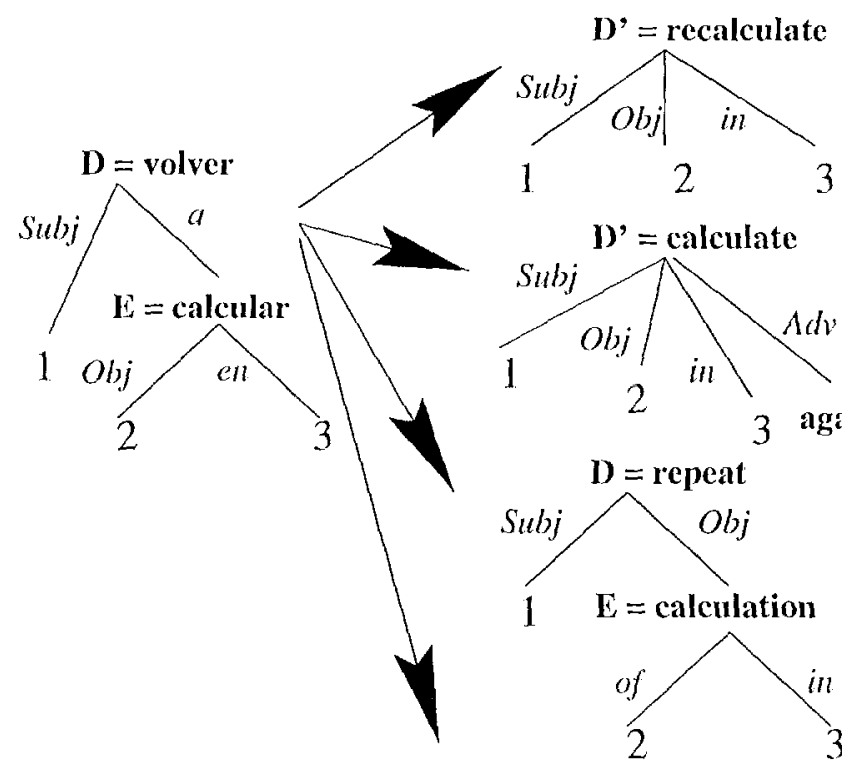

$D^{\prime}=$ calculate
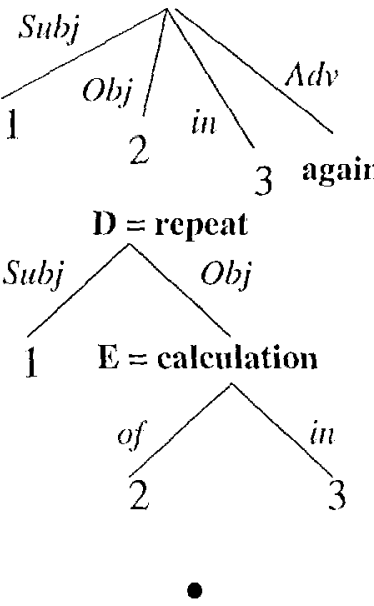

Figure 4: Multiple Transfer Rules for Each Substructure

quene prioritized by score. The procedure iteratively combines the best scoring edge with some other compatible edge to produce a new edge and inserts the new edge in the quene. The score for each new edge is a function of the scores of the edges used to produce it. The process continues until either an edge limit is reached (the system looks like it will take too long to terminate) or a complete edge is produced whose left-hand side is the input tree: we call this edge a "finished edge".

We use the following technique for calculating the score for initial edges. ${ }^{6}$ The score for each initial edge $E$ rooted at $N$, based on rule $R$ is calculated as follows:

\section{1. $\operatorname{SCORE} 1(S)=\log _{2}\left(\frac{\operatorname{Freq}(R)}{\text { Ireq(All Rules at N) }}\right)$}

Where the frequency (Freq) of a rule is the number of times it matched an example in the training corpus, during rule acquisition. The denominator is the combined frequencies of all rules that match $N$.

\footnotetext{
${ }^{6}$ This is somewhat dependent on the way these transfor rules are derived. Other systens would probably have to use some other scoring system.
}

\begin{tabular}{|l|l|l|}
\hline \multicolumn{3}{|c|}{ Ixperiment 1 : 1155 sentences } \\
\hline & Norm & No Norm \\
\hline Total Translations & 1153 & 1127 \\
Over Edge Limit & 2 & 28 \\
Actual Edges & 93,719 & 579,278 \\
Minimum Edges & 22,125 & 20,125 \\
Edge Ratio & 3.3 & 14.8 \\
Accuracy & 70.9 & 70.9 \\
\hline \multicolumn{1}{|c|}{ Lxperiment $2:$} & 2617 & sentences \\
\hline & Norm & No Norm \\
\hline Total Translations & 2610 & 2544 \\
Over Edge Limit & 7 & 73 \\
Actual Edges & 262,172 & $1,398,796$ \\
Minimum Edges & 48,570 & 42,770 \\
Edge Ratio & 4.0 & 15.5 \\
Accuracy & 62.6 & 61.5 \\
\hline
\end{tabular}

Figure 5: Resultis

\section{2. $S \operatorname{core}(S)=S \operatorname{Score} 1(S)-$ Norm Factor}

Where the Norm (normalization) factor is equal to the highest SCORE1 for any rule mat,ching $N$.

Since the $\log _{2}$ of probabilities are necessarily negative, this has the effect of setting the $E$ of each of the most probable initial edges to zero. The scores for non-initial edges are calculated by adding up the scores of the initial edges of which they are composed. ${ }^{7}$

Without any normalization $(\operatorname{Score}(S)=$ $S C O R E 1(S))$, small trees are favored over large trees. This slows down the process of finding the final result. The normalization we use insures that the most probable set of transfer rules are considered early on.

\section{Results}

Figure 5 gives our results for both experiments 1 and 2, both with normalization (Norm) and without (No Norm). "Total Translations" refor to the number of sentences which were translated successfully by the system and "Over Edge Limit" refers to the number of sentences which caused the system to exceed the edge limit, i.c., once the system produces over 10,000 edges, translation failure is assumed. The system cur-

\footnotetext{
${ }^{7}$ Scoring for special cases is not included in this paper. These cases include rules for conjunctions and rules for words that do not match any transfer rules in a given context (we currently leave the word intranslated.)
} 
rently will only fail to produce some translation for any input if the edge limit is exceeded. "Actual Edges" refers to the total number of edges used for attempting to translate every sentence in the corpus. "Minimum Edges" refer to the total minimum number of edges required for successful translations. The "Edge Ratio" is a ratio between: (1) "Total Edges" less the number of edges used in failed translations; and (2) The "Minimum Edges". This ratio, in combination with, the number of "Over Edge Limit." measures the efficiency of a given system. "Accuracy" is all assessment of translation quality which we will discuss in the next section.

Normalization caused significant speed-up for both experiments. If you compare the total number of edges used with and without normalization, speed-up is a factor of 6.2 for Experiment 1 and 5.3 for Experiment 2. If you compare actual edge ratios, speed-up is a factor of 4.5 for Experiment 1 and 3.9 for Experiment: 2. In addition, the number of failed parses went down by a factor of 10 for both experiments. As should be expected, accuracy was virtually the same with and without normalization, although normalization did cause a slight improvement. Normalization should produce the essentially the same result in less time.

These results suggest that wo can probably count on a speed-up of at least 4 and a significant decline in failed parses by using normalization. The differences in performance on the two corpora are most likely due to the degree of hand-tuning for Expcriment 1.

\subsection{Our Accuracy Measure}

"Accuracy" in Figure 5 is the average of the following score for each translated sentence:

$$
\frac{\left|T_{N Y U} \bigcap T_{M S}\right|}{1 / 2 \times\left(\left|T_{N Y U}\right|+\left|T_{M S}\right|\right)}
$$

$T_{N Y U}$ is the set of words in NYU's translation and $T_{M S}$ is the set of words in the original $\mathrm{Mi}$ crosoft translation. If $T_{N Y U}=$ "A B C D E" and $T_{M S}=$ "A B C F", then the intersection set "A B C" is length 3 (the numerator) and the average length of $T_{N Y U}$ and $T_{M S}$ is $41 / 2$ (the denominator). The accuracy score equals $3 \div 41 / 2=2 / 3$. This is a Dice coefficient comparison of our translation with the original. It is an inexpensive method of measuring the perfor- mance of a new version of our system. Improvements in the average accuracy score for our sample set of sentences usually reflect an improvement in overall translation quality. While it is significant that the accuracy scores in Figure 5 did not go down when we normalized the scores, the slight improvement in accuracy should not be given much weight. Our accuracy scorc is flawed in that it cannot account for the following facts: (1) good paraphrases are perfectly acceptable; (2) some differences in word selection are more significant than others; and (3) crrors in syntax are not directly accounted for.

NYU's system translates the Spanish sentence "1. Seleccion la celda en la que desea introducir una referencia" as "1. select the cel1 that you want to enter a reference in". Microsoft translates this sentence as "1. Select the cell in which you want to enter the reference". Our system gives NYU's translation an accuracy score of .75 due to the degree of overlap with Microsoft's translation. A human reviewer would probably rate NYU's translation as completely acceptable. In contrast, NYU's system produced the following unacceptable translation which also received a score of .75 : the Spanish sentence "Elija la función que desea pegar en la fórmula en el cuadro de diálogo Asistente para funciones" is translated as "Choose the function that wants to paste Function Wizard in tho formula in the dialog box", in contrast with Microsoft's translation "Choose the function you want to paste into the formula from the Function Wizard dialog box". In fact, some good translations will get worse scores than some bad ones, e.g., an acceptable one word translation can even get a score of 0 , e.g., "SUPR" was translated as "DEL" by Microsoft and as "Delete" by NYU. Nevertheless, by averaging this accuracy score over many examples, it has proved a valuable measure for comparing different versions of a particular system: better systems get better results. Similarly, after tweaking the system, a better translation of a particular sentence will usually yield a better score.

\section{Future Work}

Future work should address two limitations of our current system: (1) Bad parses yicld bad transfer rules; and (2) sparse data limits the size of our transfer rule database and our options for 
applying transfer rules selectively. To attack the "bad parse" problem, we are considering using our MT system with less-detailed parsers, since these parsers typically produce less error-prono output. We will have to conduct experiments to determine the minimum level of detail that is needed. ${ }^{8}$

Previous to the work reported in this paper, we ran our MT system on bilingual corpora in which the sentences were aligned manually. The cost of manual alignment limited the size of the corpora we could use. A lot of our recent MT research has been focused on solving this sparso data problem through our devclopment of a sentence alignment program (Meycrs et al., 1998a). We now have 300,000 automatically aligned sentences in the Microsoft help text domain for future experiments. In addition to providing us with many more transfer rules, this should allow us to collect transfer rule co-occurrence information which we can then use to apply transfer rules more effectively, perhaps improving translation quality. In a preliminary experiment along these lines using the Experiment 1 corpus, co-occurrence information had no noticeable offect. However, we are hopeful that future experiments with 300,000 aligned sentences (300 times as much clatia) will be more suceessful.

\section{References}

Robert J. Bobrow. 1990. Statistical agenda parsing. In DARPA Speech and Language Workshop, pages 222-224.

Peter Brown, Stephen A. Della Pietra, Vincent J. Dolla l'ietra, and Robert L. Morcer. 1993. The Mathematics of Statistical Machine Translation: Paranneter Estimation. Computational Linguistics, 19:263-312.

Sharon A. Caraballo and Eugene Charniak. 1997. New figures of morit for best-first probabilistic chart parsing. Computational Linguistics, 24:275-298.

Eugene Charniak, Sharon Goldwater, and Mark Johnson. 1998. Edge-Based Best-First Chart, Parsing. In Proceedings of the Sixth Annual Workshop for Very Large Corpora, Montrcal.

\footnotetext{
${ }^{8}$ One could set up a continuum from cletailed parser$s$ like Proteus down to shallow verb-group/noun-group recognizers, with the Penn treebank based parsers lying somowhere in the middle. As one travels down the contintum to the lower detail parsers, the error rate naturally decreases.
}

Mahesh V. Chitano and Ralph Grishman. 1990. Statistical parsing of messages. In DARPA Specch and Language Workshop, pages 263 266.

Amla Sågvall Hein. 1996. Preference Mochanisms of the Multra Machine Translation Systom. In Barbara H. Partee and Petr Sgall, editors, Discourse and Meaning: Papers in Honor of Lva Hajiçová. John Benjamins Publishing Company, Amsterdam.

Y. Matsumoto, H. Ishimoto, T. Utsuro, and M. Nagao. 1993. Structural Matching of Parallel Texts. In 31st Annual Mecting of the Association for Computational Linguistics: "Proceedings of the Conference".

Adam Meycrs, Roman Yangarber, and Ralph Grishman. 1996. Alignmont of Shared Forests for Bilingual Corpora. In Proceedings of Coling 1996: The 16th International Conference on Compulational Linguistics, pages $460-465$.

Adam Mcycrs, Michiko Kosaka, and Ralph Grishman. 1998a. A Multilingual Procedure for Dictionary-Based Sentence Alignment. In Procedings of AMTA'98: Machine Translation and the Information Soup, pages 187198.

Arlam Meyers, Roman Yangarber, Ralph Grishman, Catherine Macleod, and Antonio Moreno-Sandoval. 1998b. Deriving Trans-

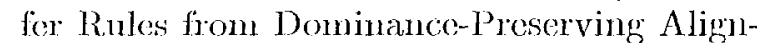
ments. In Procedings of Coling-ACL98: The $1^{7} 7$ th International Conference on Computational Linguistics and the 36th Mecting of the Association for Computational Linguistics.

Sergei Nirenburg and Robert E. Troderking. 1995. The Pangloss Mark III Machine Thanslation System: Multi-Engine System Architecture. 'Jechnical report, NMSU CRL, USC ISI, and CMU CMT.

Andrew Way, Ian Crookston, and Jane Shelton. 1997. A Typology of Translation Problems for Eurotra Thanslation Machines. Machine Translation, 12:323 374 . 